\title{
Transverse motions of a single sphere in hanging or swing configurations under a longitudinal flow
}

\author{
Mikel Ogueta-Gutiêrrez, Omar Gómez-Ortega, Sebastiân Franchini, \\ Angel Sanz-Andres*, Javier Perez-Alvarez, Fernando Meseguer-Garrido \\ IDR/UPM, Universidad Politécnica de Madrid, Pza, del Cardenal Cisneros 3, 28040 Madrid, Spain
}

\section{A R T ICL E I N F O}

\section{Article history:}

Received 5 May 2018

Received in revised form 4 November 2018

Accepted 14 November 2018

Available online 28 November 2018

\begin{abstract}
A B S T R A C T
As a result of the reporting of casual observations of the oscillation or rotation of the beacons in transmission line guard cables, some attention has been paid to the stability of the guard cables with beacons.

The relatively more frequent observation of these motions has been explained in recent papers dealing with the elastic part of the problem as a consequence of the increasing number of resonant frequencies (one for each additional beacon) that can be excited by appropriate aerodynamic loads. But a model that could explain the aerodynamic forces that can give rise to this motion is still lacking.

In this paper we consider the transverse motions of a single sphere in two simplified configurations, (1) hanging (tethered at one point), and (2) swing (tethered at two points) under a longitudinal flow, performing small amplitude swinging oscillations or circularorbit autorotation about an axis parallel to the main flow direction. The dynamic model here presented is based on the motion equations, which also include a model for the aerodynamic lift and drag forces on the sphere in transverse motion, which consider's the effect of changes of flow around the sphere due to the cable interference. These forces are contained in the symmetry plane of the flow relative to the sphere, and, when projected on the lateral direction, give tise to a lateral force, which can explain the existence of the azimuthal motion even at a large reduced velocity, outside the vortex induced vibration (VIV) range The conditions for stable small oscillation motion and circular-orbit autorotation of a sphere in a swing configuration are given.

The results for the aerodynamic loads in transverse motion have also been applied to the case of a circulat-orbit autorotation of a hanging sphere (spherical pendulum) under a vertical flow. The angular rotation speed and the orbit radius (or cable angle) have been determined as a function of aerodynamic coefficients and configuration parameters.
\end{abstract}

(c) 2018 Elsevier ltd. All rights reserved.

\section{Introduction}

Some attention has been paid to the stability of the transmission line guard cables with beacons as a result of reports of casual observations of the oscillation or whirling motions of the beacons in some transmission lines (Gómez-Ortega et al., 2019).

Guard cables protect the overhead power lines from electrical discharges that occur during storms. They are installed above the power conductor and therefore, they are the highest cable installed. In some places they are provided with 


\begin{tabular}{|c|c|}
\hline \multicolumn{2}{|l|}{ Nomenclature } \\
\hline$A$ & Angle between the symmetry plane $\Pi$ and the horizontal plane \\
\hline$C_{l}$ & Lift coefficient \\
\hline$C_{D}$ & Drag coefficient \\
\hline$C_{D 0}$ & Drag coefficient at $\gamma=0$ \\
\hline$C_{F y b}$ & Lateral force coefficient \\
\hline$C_{L y}$ & Slope of the lift coefficient curve \\
\hline$C_{H}$ & Den Hartog parameter \\
\hline$D$ & Drag \\
\hline$d_{B}$ & Beacon diameter \\
\hline$F_{b}\left\{O_{b}, x_{b}, y_{b}, z_{b}\right\}$ & Body reference frame \\
\hline$F_{h}\left\{O_{h}, x_{h}, y_{h}, z_{h}\right\}$ & Local horizon reference frame \\
\hline$F_{I}$ & Lateral force \\
\hline$F_{y b}$ & Lateral force component along $\mathbf{e}_{y b}$ \\
\hline $\mathrm{Fr}$ & Froude number \\
\hline \multicolumn{2}{|c|}{$F_{u^{\prime}}\left\{\mathrm{O}_{w}, x_{u^{\prime}}, y_{w}, z_{u^{\prime}}\right\}$ Wind reference frame } \\
\hline$f_{R}$ & Natural frequency \\
\hline$g$ & Gravity acceleration \\
\hline$K_{\delta}$ & Cable angle parameter \\
\hline$K_{L}$ & Tachikawa number, load factor \\
\hline$L$ & Lift \\
\hline 1 & Cable length \\
\hline $\mathfrak{m}$ & Mass \\
\hline $\mathfrak{m}_{d}$ & Displaced mass \\
\hline$m_{n}$ & Moving mass \\
\hline$m^{*}$ & Reduced mass \\
\hline$q_{\infty}$ & Dynamic pressure \\
\hline $\operatorname{Re}$ & Reynolds number \\
\hline $\mathrm{Re}_{\mathrm{c}}$ & Critical Reynolds number \\
\hline$r_{z}$ & Rotation radius, static equilibrium sag \\
\hline $\mathrm{r}_{p}$ & Participation coefficient \\
\hline$S_{j}$ & Sphere reference surface area \\
\hline t & Time \\
\hline$U_{\infty}$ & Unperturbed flow velocity \\
\hline$U_{0, y}$ & Relative wind speed \\
\hline$y$ & Lateral position \\
\hline$Y(t)$ & Dimensionless transverse position \\
\hline$Y_{0}(\omega)$ & Complex amplitude \\
\hline$\beta$ & Sideslip angle \\
\hline$\delta_{c}$ & Static cable angle \\
\hline$\varepsilon_{8}$ & Dimensionless shift of the rotation angular speed \\
\hline$\varepsilon_{1}$ & Ancillary dimensionless aerodynamic parameter \\
\hline$\gamma$ & Dynamic cable angle \\
\hline$\omega_{d t}$ & Dimensionless angular speed \\
\hline$\omega_{y}$ & Characteristic angular speed \\
\hline$\Omega$ & Angular rotation speed \\
\hline$\Omega_{0}$ & Rotation equilibrium speed in absence of aerodynamic forces \\
\hline$\Pi$ & Symmetry plane for the relative fluid flow \\
\hline$\rho$ & Density \\
\hline$\zeta$ & Damping coefficient \\
\hline$\tau$ & Dimensionless time \\
\hline
\end{tabular}




\section{Subscripts}

\begin{tabular}{ll} 
ar & Air \\
ar & Autorotation \\
$B$ & Beacon \\
$C$ & Cable \\
$E$ & Experimental \\
$M$ & Mathematical model \\
0 & Oscillation \\
$x, y, z, x h, y h, z h$, & Components along the respective axes \\
$x w, y w, z w, y w \pi$ & \\
\hline
\end{tabular}

beacon spheres that are visible indicators used for identifying the overhead power lines and to warn low flying airplanes and helicopters of the obstructions.

The interest behind the study in this paper is to understand the transverse instability that appears in guard cables with beacons under a longitudinal wind, as reported by Gómez-Ortega et al. (2019), which is the first published paper devoted to this problem, and precursor of the work presented here. This is a very complex problem as the number of degrees of freedom increases with the number of beacons, which gives rise to a corresponding growth in the number of eigenfrequencies of the elastic problem. In order to ease the understanding of the problem, attention has been paid to the simplified case of a single sphere, oscillating or revolving in circular orbits about an eccentric axis (the support line which does not coincide with the sphere central axis) in this case forced by a longitudinal flow (parallel to the rotation axis). The study of the problem of a cable with multiple spheres is left for a future paper.

The aim of this paper is to present a model of aerodynamic forces and of the flight dynamic problem that can help to explain the transverse motion of oscillation or autorotation of spheres (tethered in several configurations), under a longitudinal flow. One of the main parameters of the configuration is the static cable angle $\delta_{c}$ between the cable and the flow direction. Some of these configurations exhibit motions that can be denoted as circular orbits and others are oscillatory motions (small amplitude swinging in the lower part of the circular trajectory) (Fig. 1):

a-The hanging sphere (as a spherical pendulum) under a vertical flow (Provansal et al., 2004). The static cable angle is $\delta_{c}<20^{\circ}$. Both gravity force and fluid motion are in a vertical direction. The body center can describe planar, elliptical and circular orbits, although only this last case is considered here.

b-The single sphere in a swing configuration, under a flow that is directed along the swing support line direction SS, $x_{h}$, perpendicular to the gravity force direction, $z_{h}$. The cable angle range of interest is $\delta_{c}<20^{\circ}$ (Gómez-Ortega, 2018). The sphere center point can perform full turns of the circular orbit (autorotation) or small amplitude oscillations sweeping the lower part of the circular orbit. We consider here both cases.

c-Multiple spheres in a swing configuration (as in a guard cable with several beacons) in the same conditions as the single sphere (see pictures in Gómez-Ortega et al., 2019). This problem is not considered here.

In the case of a single sphere in a swing configuration (Fig. 1b), the trajectory could either cover the full circular orbit (autorotation) or just a small angle (small amplitude swinging oscillations).

Other configurations, such as the tethered spherical buoy (Fig. 1d), instead of a circular orbit, exhibit a figure-eight orbit (Govardhan and Williamson, 2005; Williamson and Govardhan, 1997, 2008). The sphere is hanging or buoying in a fluid with a different density, and moves in a horizontal flow stream, under the action of the gravity force, cable tension, and aerodynamic forces. In a general case, due to density difference, the static cable angle is $\delta_{c}>60^{\circ}$. The above mentioned $20^{\circ}$ and $60^{\circ}$ limits are merely indicative of small or large cable angle values.

In all these motions a transverse aerodynamic force is responsible for starting and/or maintaining the motion. Such a force should act in the appropriate direction; otherwise it would stop or dampen the sphere motion.

The cable tension and the line sag are both related to the static cable angle $\delta_{c}$ (if $\delta_{c}$ is small, sag is also small and tension should be large). The type of motion that appears can be associated to the relative value of the tension compared to the aerodynamic force, and the orientation of the buoyancy/gravity forces, leading to either circular or figure-of-eight orbits.

In all cases the motion is characterized by a transverse motion of the sphere, which combined with the main flow gives rise to a sideslip angle of the flow relative to the sphere (see Fig. 2).

If a bluff body in a flow is precisely spherical, then a change in the angle of attack of the flow produces no change in the aerodynamic forces because of the spherical symmetry. However, when the bluff body has a tether with the nominal flow velocity parallel to the tether, and the body is displaced laterally, the spherical symmetry is lost because of the tether wake.

In a precisely spherical bluff body, without the tether, the sideslip angle cannot be defined due to the lack of material references, caused by the sphere isotropy. However, in the above mentioned configurations the sphere is supported by cables, which introduce a perturbation that defines a material reference. This reference, together with the flow direction, defines a symmetry meridian plane, but then isotropy is lost. If there is a transverse displacement with regard to this symmetry plane, a sideslip angle appears.

The aerodynamic force on a sphere, with a cable placed upstream at a given angle $\delta_{c}$ with the flow direction, can be studied as a quasi-static problem, in the appropriate speed range (where a quasi-steady aerodynamic flow can be considered), that 




(a)

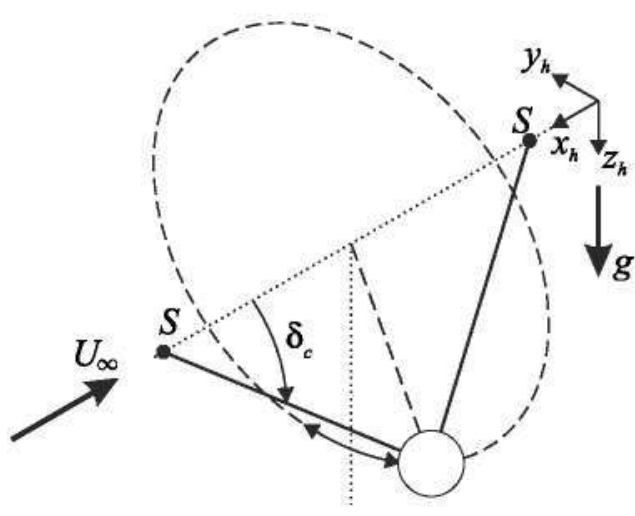

(b)
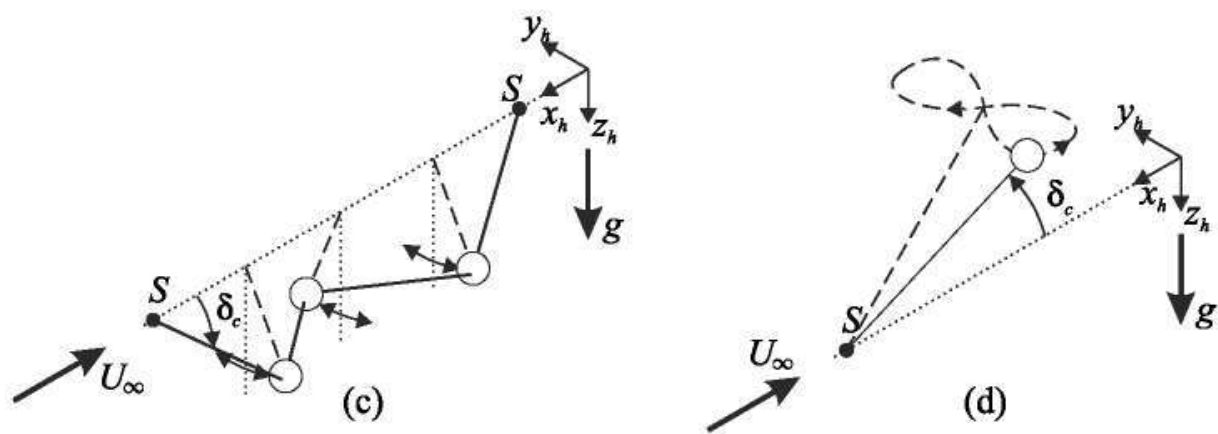

Fig. 1. Sketch of some configurations that can exhibit full and/or partial circular orbits: (a) hanging sphere, tethered at support S, under a vertical flow $U_{\infty}$. (full circular-orbit autorotation); (b) single sphere in a swing configuration, tethered at two supports $S$, under a horizontal flow, it can exhibit both small amplitude pendulum oscillations and full circular orbit motions (autorotation); (c) multiple spheres performing horizontal oscillations in a swing configuration, under a horizontal flow. Figure-of-eight orbits: (d) tethered spherical buoy. $\delta_{c}$ : static cable angle; S: support point; g: gravity force direction along $z_{\hbar}$ axis. Incoming flow $U_{\infty}$.

is, out of the unsteady VIV range. This is valid for both the oscillation or autorotation motions if the transverse speed is not too large, as far as the cable wake can be considered almost like a plane.

The aerodynamic force on a static sphere, in steady flow conditions, is a classical fluid mechanical problem and has been experimentally studied for a long time (Achenbach, 1972, 1974a,b; Willmarth and Enlow, 1969). At first sight, the sphere seems to be a simple body that can be used as a standard or reference body for wind tunnel performance comparisons. But its simplicity and isotropy create some curious problems. Noticeable discrepancies concerning the results for drag coefficient $C_{D}$ measured in several wind tunnels were reported when the different results were compared by Bacon and Reid (1924) in an investigation to explain the origin of these discrepancies, in which the effect of the supporting system and the turbulence level were taken into account. Relevant for the current paper, it was shown that the spindle or the orientation of the supporting wire can have a large influence in the $C_{D}$ measured. The effect of the surface roughness and wind tunnel section blockage (Achenbach, 1974b; Choi et al., 2006) are also relevant.

With the aim of studying the possibility of using morphing techniques to control the forces on a sphere (Norman and McKeon, 2011a,b) devoted their attention to measure the forces and the associated flow characteristics on a carefully-built smooth sphere, both with and without a single roughness element of small size. In the case of the smooth sphere, they found that the symmetry of the flow can be destroyed by very small perturbations, which in a practical situation are very difficult to get rid of. They tried studs (circular cylinders of the same diameter as height) whose size were $1 \%, 2 \%$ and $4 \%$ of the sphere diameter. Even with these small sizes, they were able to generate lift forces of the same order of magnitude as drag.

The effects of the Reynolds number, Re, and the stud size and longitudinal position along the sphere surface (streamwise angle) were also considered by these authors. The critical Reynolds number, $\operatorname{Re}_{c}$, is defined by these authors as the value 
at which the sign of the lift force changes, and is close to the classical definition of the value at which a large change in drag coefficient is observed (drag crisis). Interestingly, the lift force is in the direction of the stud in the subcritical range but changes sign in the supercritical range, due to a change in the flow configuration involved. If we consider the sphere as a small span wing, if the perturbation is placed in the lower surface the wing gives positive lift $(L>0)$ in the supercritical range ( $\operatorname{Re}>\operatorname{Re}_{c}$ ), and negative lift $L<0$ in the subcritical range ( $\operatorname{Re}<\operatorname{Re}_{c}$ ). The reason is that the flow changes from the generation of vortexes at the protrusion (that attaches the boundary layer in the leeward side of the protrusion for $\operatorname{Re}<\operatorname{Re}_{c}$ ) to a configuration at $\operatorname{Re}>\operatorname{Re}_{c}$ with the boundary layer detached at the protrusion, thus spoiling the lift distribution of the sphere surface affected by the protrusion, and generating positive lift.

In Norman et al. (2011) the azimuthal motion of the stud is considered. One of the relevant results is that in the subcritical range the lift force can reach a maximum value similar to $C_{D}$ at an appropriate rotation speed, which is a subharmonic of the vortex shedding frequency, associated with a local perturbation of the separation location. In the supercritical range the lift force is $180^{\circ}$ out of phase with regard to the stud position, as in the static case. In the subcritical range, at a low azimuthal rotation speed, the lift force is in phase with the stud position, but for a large rotation speed the lift force also reaches the $180^{\circ}$ out of phase as in the supercritical range. It can be concluded that the effect of the cable or supporting wires cannot be neglected when analyzing the motion of spheres supported with wires. And the lift force is always positive ( $180^{\circ}$ out of phase with the perturbation) even in the subcritical range once the rotation rate has reached a given value.

In addition to oscillation, autorotation is another of the possible motions (see Fig. 1) of both a spherical pendulum (hanging sphere where both flow and gravity are in vertical direction) and of a planar pendulum (spheres in a swing configuration where flow and gravity are in perpendicular directions). Sometimes, autorotation is considered as any continuous rotation of a body in a parallel flow without external sources of energy, and whenever these bodies are kept fixed in a fluid flow, a torque is created that initiates rotation as soon as the bodies are released. But according to Lugt (1983) proper autorotation appears, in the sense of Riabouchinsky (1935), "only if one or more stable positions exist at which the fluid flow exerts no torque on the resting body. In this case, a sufficiently strong initial impulse is required before the fluid flow can sustain a continuous spinning of the body". In the case of a sphere, turbulence or other flow perturbation could produce this initial impulse.

Two simple configurations are considered here: (1) a single hanging sphere (tethered at one support point) under a vertical flow (Fig. 1a), and (2) the swing configuration (the sphere is tethered at two supports by two opposite cables) under a horizontal flow (Fig. 1b). The mainstream direction changes from parallel to perpendicular to the gravity force direction. The common point to both configurations is that the aerodynamic load can be described by the same model: quasi-steady lift and drag forces created by the interaction of the cable wake with the sphere surface; these forces are placed in the symmetry plane of the flow relative to the moving sphere. This relative symmetry plane is not perpendicular to the transverse direction, thus, the projection of the aerodynamic load gives rise to a transverse force that helps to produce or maintain the motion.

Therefore, the motions considered share the way of dealing with the aerodynamic forces, and the mechanical problems considered (small amplitude oscillation and circular autorotation) have simple dynamic descriptions. This situation greatly reduces the complexity of the problems, and, together with some asymptotic approximations, allows us to obtain some analytical expressions for the problem solutions, which help to identify and assess the influence of the parameters involved.

Following this approach, in Section 2 a model of the aerodynamic loads acting on the sphere, including the interaction with the cable wake, is presented, together with the relevant reference frames required, and the main assumptions considered to simplify the formulation and facilitate the analysis. Several flight dynamic problems based in this aerodynamic model are considered: for a single sphere in a swing configuration performing a small amplitude oscillation motion in Section 3 , and for a circular-orbit autorotation motion in Section 4; and the same autorotation motion for a hanging sphere is considered in Section 5; and finally in Section 6 conclusions are drawn.

\section{Aerodynamic forces on a cable-supported sphere in transverse motion}

In this Section, a model to determine the aerodynamic loads on a sphere that is performing a transverse motion under a parallel flow is presented, taking into account the effect of the cable wake. A quasi-steady flow approximation is considered.

\subsection{Introduction and reference frames}

In the case of a sphere that is free to revolve around an eccentric axis, and is placed under a longitudinal flow parallel to the rotation axis, it can exhibit pendulum oscillations or orbit autorotation if there is a lateral aerodynamic force generating a torque that excites the pendulum motion or maintains the orbit motion, respectively. For instance, in the case of a guard cable with only one beacon (single sphere in a swing configuration, Fig. 1b) under a large flow velocity (nearly parallel to the main flow) in the autorotation motion the beacon performs a circular motion around the eccentric axis (the straight line passing through the cable support points, line SS). This motion can be considered as a steady one, if considered in a reference frame fixed to the sphere. In the case of low frequency, small amplitude oscillations, the flow can be considered as quasi-steady, and the results of this section can also be applied.

The calculation of the aerodynamic force involved in the motion can be complex. In order to simplify this formulation, we consider the configuration that appears at a particular time instant, and assume that the body is instantaneously moving in a straight trajectory along the tangential direction. Thus, we consider both the oscillatory and circular motions as a combination of differential tangential straight motions. 
Therefore, for the simplification of the representation, we consider that the body is performing an instantaneous rectilinear motion along the transverse direction, and choose the point of the trajectory where this rectilinear motion can be represented by one of the reference frame axis $y_{b}$ (Fig. 2). This point corresponds to the time instant when the body passes through the upper point in its circular trajectory, but the results can be applied to determine the transverse force at any time instant of the motion, even in the case of oscillations. This specific time instant has been selected because it brings one of the best perspectives to show the relative position of the reference frames and of the spherical triangle $\beta-\gamma-\delta_{\mathrm{c}}$. The existence of this triangle is the base of the model and the reason for the presence of the lateral force that is responsible for the motion.

In the description of the forces acting on the body we will consider three reference frames, as is usual in some problems of Flight Dynamics (Etkin, 1972). These reference frames are shown in Fig. 2, where the main directions are represented, with the help of the unit radius sphere (Wertz and Larson, 1999). This sphere is used to help in the geometrical definition of the different angles involved in the problem and should not be confused with the beacon itself, which in this figure is just a mass point placed at the origin of the body reference frame. The reference frames considered here are the following ones:

-Local horizon reference frame: $F_{h}\left\{\mathrm{O}_{h}, x_{h}, y_{h}, z_{h}\right\}$, where $O_{h}$ is placed at the rotation axis, which in the time instant considered is just below the body center of mass $O_{b} ; x_{h}$ is the rotation axis, placed in the horizontal plane, aligned with both the support line direction and the unperturbed flow direction $U_{\infty} . z_{h}$ is directed along the local vertical. This system is considered as an inertial reference frame.

-Body reference frame: $F_{b}\left\{\mathrm{O}_{b}, x_{b}, y_{b}, z_{b}\right\}$, where $O_{b}$ is placed at the body center of mass; $x_{b}$ is parallel to $x_{h}$ at every instant; $y_{b}$ and $z_{b}$ are parallel to $y_{h}$ and $z_{h}$, respectively, at the time instant considered. The distance between $O_{b}$ and $O_{h}$ is the rotation radius $r_{g}=\overline{\mathrm{O}_{h} \mathrm{O}_{b}}$.

-Wind reference frame: $F_{w}\left\{\mathrm{O}_{w}, x_{w}, y_{w}, z_{w}\right\}$, where $O_{w}$ coincides with $O_{b} ; x_{w}$ is aligned with the relative wind speed $U_{\infty}$; and $\beta$ is the sideslip angle. $z_{w}$ and $z_{h}$ are parallel to each other at the time instant considered.

A meridian symmetry plane $\left(x_{b}, z_{b}\right)$ can be defined in the sphere that contains the cable direction $\mathrm{O}_{b} \mathrm{C}$ and the sphere axis parallel to the unperturbed flow $x_{b}$. The static cable angle $\delta_{c}$ is contained in this plane.

But to determine the aerodynamic force a different symmetry plane should be considered. Let us consider the relative flow velocity $U_{\infty r}$, which is the composition of the uniform unperturbed flow velocity, $U_{\infty}$ (along $x_{b}$ axis) and a lateral component of the motion speed along $y_{b}, \Omega r_{z}$, produced by the angular rotation speed $\Omega$ (Fig. 3). The incident flow velocity is not uniform but to simplify the problem we will consider a uniform relative velocity $U_{\infty r}=\sqrt{U_{\infty}^{2}+\Omega^{2} r_{g}^{2}}=U_{\infty} \sqrt{1+\tan ^{2} \beta}$ where $\beta=\operatorname{atan}\left(\Omega r_{g} / U_{\infty}\right)$.

In a general case an angle of attack can also appear, but here only the sideslip angle $\beta$ is considered. Rotation around axis $x_{h}$ is transformed in an instantaneous sideslip motion in direction $y_{b}$. At the time instant considered, moving reference frame $F_{b}$ and inertial frame $F_{h}$ are parallel to each other.

This is just a simplified way of defining the tangential motion, analyzing only one point of the trajectory, the passing by the highest point of the circular motion in the autorotation case.

\subsection{Aerodynamic forces. Quasi-steady model}

As a consequence of the transverse motion (Fig. 3), a relative lateral velocity component should be taken into account, which gives rise to the relative wind velocity, $U_{\infty}$, and the sideslip angle $\beta$. The combination of static cable angle, $\delta_{c}$, and sideslip angle, $\beta$, leads to the dynamic cable angle $\gamma$ defined as the angle between the relative wind velocity, $U_{\infty r}$, and the cable direction. Cable direction $C$ and relative velocity $U_{\infty}$ define a symmetry plane $x_{w}, y_{w \pi}$ (denoted as $\Pi$ ) for the relative fluid flow (Fig. 4). From symmetry considerations it can be deduced that, under quasi-steady conditions, the net aerodynamic force will be within this plane (as the lateral force normal to plane $\Pi$ is zero, by symmetry). Therefore, the aerodynamic force components are only lift, $L \mathbf{e}_{y w \pi}$, and drag, $-D \mathbf{e}_{x w}$.

To study the beacon dynamics, the force component along the motion direction $y_{b}$ should be determined. The component of $\mathbf{e}_{y w \pi}$ along $\mathbf{e}_{y w}$ (Figs. 4 and 5) can be obtained from

$$
\mathbf{e}_{y w \pi}=\mathbf{e}_{y w} \cos A-\mathbf{e}_{z b} \sin A
$$

where $A$ is the angle between the symmetry plane $\Pi$ and the horizontal plane. The component of the net aerodynamic force $\left(L \mathbf{e}_{y w \pi},-D \mathbf{e}_{x w}\right)$ on the plane $x_{w}, y_{w}$ is given by

$$
\mathbf{F}_{x y w}=L \mathbf{e}_{y w} \cos A-D \mathbf{e}_{x w}=L \cos A\left(-\mathbf{e}_{x b} \sin \beta+\mathbf{e}_{y b} \cos \beta\right)-D\left(\mathbf{e}_{x b} \cos \beta+\mathbf{e}_{y b} \sin \beta\right)
$$

as

$$
\begin{aligned}
& \mathbf{e}_{x w}=\mathbf{e}_{x b} \cos \beta+\mathbf{e}_{y b} \sin \beta \\
& \mathbf{e}_{y w}=-\mathbf{e}_{x b} \sin \beta+\mathbf{e}_{y b} \cos \beta
\end{aligned}
$$

Therefore

$$
\mathbf{F}_{x y w}=\mathbf{e}_{x b}(-L \cos A \sin \beta-D \cos \beta)+\mathbf{e}_{y b}(L \cos A \cos \beta-D \sin \beta)
$$




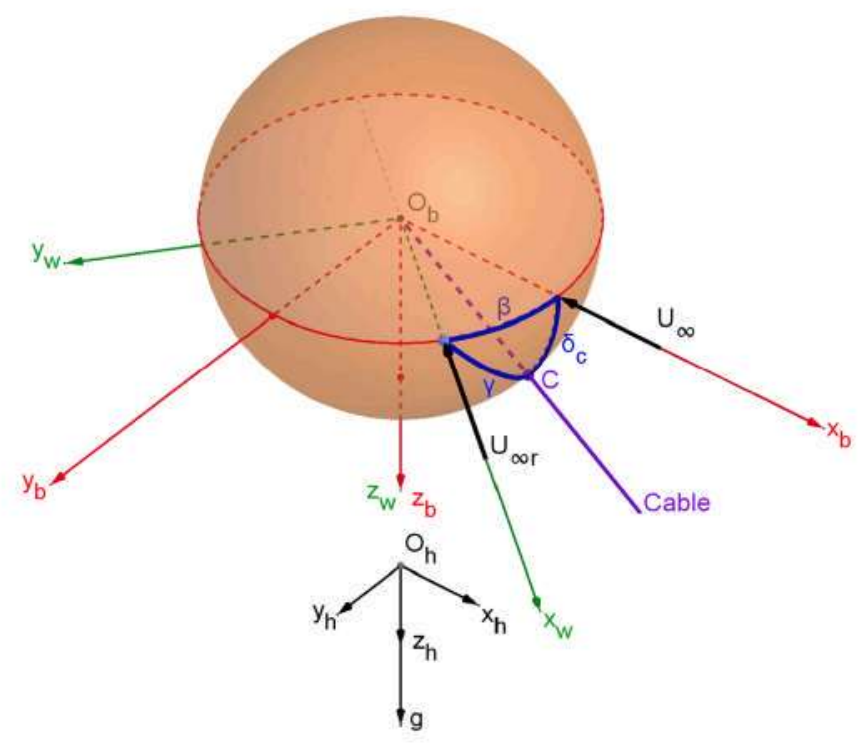

Fig. 2. Reference frames considered: local horizon $F_{h}\left\{O_{h}, x_{h}, y_{h}, z_{h}\right\}$ (in black), body $F_{b}\left\{O_{b}, x_{b}, y_{b}, z_{b}\right\}$ (in red), and wind $F_{w}\left\{O_{w}, x_{w}, y_{w}, z_{w}\right\}$ (in green) reference systems, respectively. $\beta$ : sideslip; $\delta_{c}$ : static cable angle; $\gamma$ : dynamic cable angle; $\mathrm{C}$ : cable direction (only the windward cable is shown); $U_{\infty}$ : unperturbed flow velocity; $U_{c o r}$ : relative flow velocity. Angles are represented using the unit radius sphere (colored sphere). g: gravity force direction. Axonometric projection.

The effect of the horizontal force component along $\mathbf{e}_{x b}$ is to change the cable tension $T_{0}$. However, as the tension changes due to drag effect are negligible $\left(T_{0} \gg L, D\right)$ this component is not further considered. The torque around the rotation axis $x_{h}$ is produced by the lateral force component along $\mathbf{e}_{y b}$ given by

$$
F_{y b}=L \cos A \cos \beta-D \sin \beta
$$

which can speed up or slow down the motion, depending on the sign. From spherical geometry (Wertz and Larson, 1999), see Fig, 6,

$$
\cos \gamma=\cos \delta_{c} \cos \beta \quad \tan A=\frac{\tan \delta_{c}}{\sin \beta}
$$

where $\gamma$ is the dynamic cable angle for the relative flow. In a typical case the angles can be considered to cover a small range $\delta_{c}, \beta \ll 1$ and therefore $\gamma \ll 1$. The first expression can be expanded to give

$$
1-\frac{\gamma^{2}}{2} \simeq\left(1-\frac{\delta_{c}^{2}}{2}\right)\left(1-\frac{\beta^{2}}{2}\right) ; \gamma^{2} \simeq \beta^{2}+\delta_{c}^{2}
$$

and from the second it gives $\tan A \simeq \delta_{c} / \beta$, that can be further simplified, if $\delta_{c} \ll \beta \ll 1$

$$
\tan A \simeq A \simeq \frac{\delta_{c}}{\beta}
$$

The lateral force coefficient can be defined as

$$
C_{F y b}=\frac{F_{y b}}{q_{\infty} S_{S}}=C_{L} \cos A \cos \beta-C_{D} \sin \beta
$$

where $q_{\infty}$ is the dynamic pressure, and $S_{S}$ is the sphere reference surface area for the aerodynamic forces. $C_{L}$ and $C_{D}$ are the lift and drag coefficients of a sphere with a cable placed at an angle $\gamma$ with regard to the relative incoming flow direction

$$
C_{L}=C_{L}(\gamma) \quad C_{D}=C_{D}(\gamma)
$$

In the case $\delta_{c}, \beta \ll 1$

$$
\begin{aligned}
& \cos A=\frac{1}{\sqrt{1+\tan ^{2} A}} \simeq \frac{1}{\sqrt{1+\left(\delta_{c} / \beta\right)^{2}}}=\frac{\beta}{\sqrt{\beta^{2}+\delta_{c}^{2}}}=\frac{\beta}{\gamma} \\
& C_{F y b}=C_{L} \frac{\beta}{\gamma}-C_{D} \beta=\beta\left(\frac{C_{L}}{\gamma}-C_{D}\right)
\end{aligned}
$$


a)
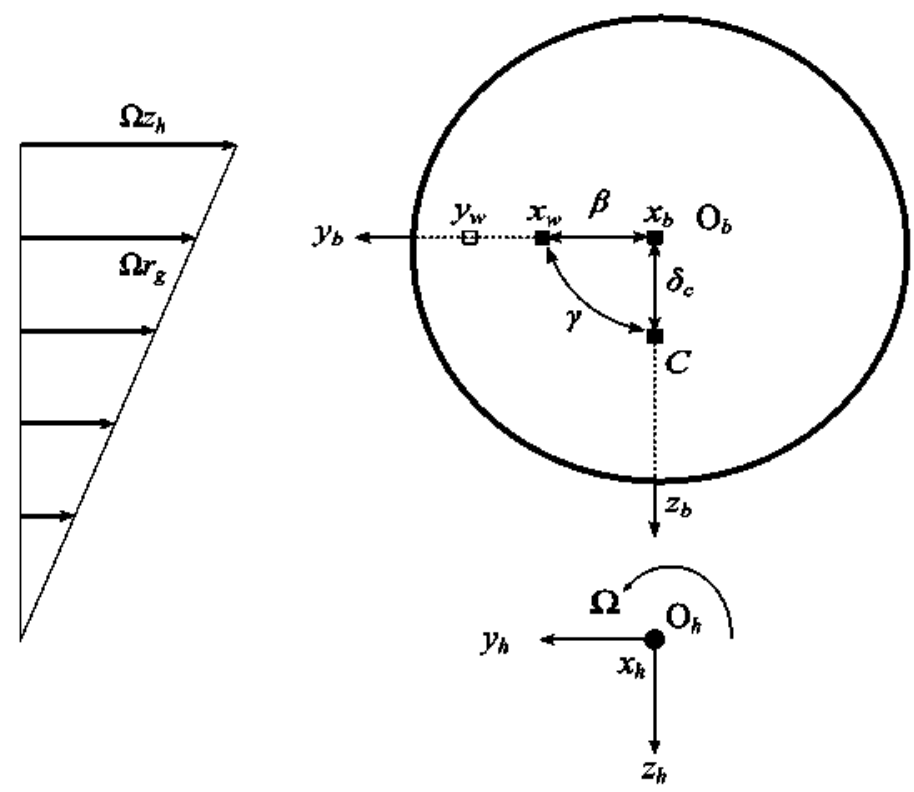

b)

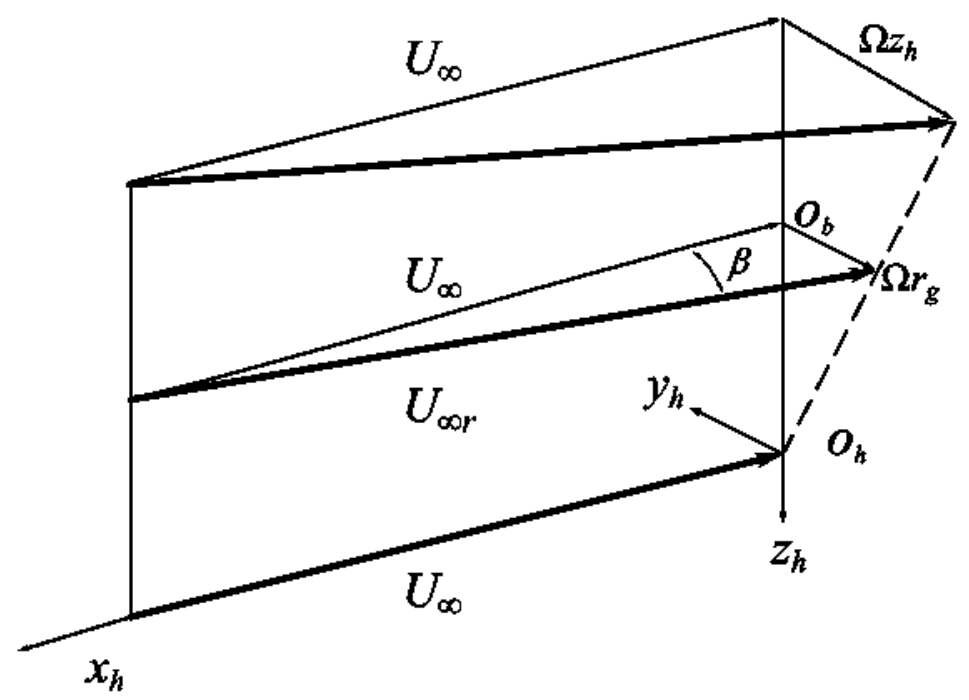

Fig. 3. (a) Projection on the transverse plane $y_{b}, z_{b}$ of the unit sphere directions and the incoming relative flow speed. $\beta:$ sideslip: $\delta_{c}$ : static cable angle: $\gamma$ : dynamic cable angle. The beacon center is revolving around axis $x_{h}$ (point $\mathrm{O}_{\mathrm{h}}$ ) at speed $\Omega$. The sketch corresponds to the time instant considered in the analysis. Position of the intersection points with the unit sphere surface; with cable direction $C$ and $x_{b}$ and $x_{w ;}$ axes (foreground, full square), and $y_{t c}$ (background, empty square). (b) Velocity compositions at different distances from the rotation axis $x_{k}$. At distance $r_{g}$ : the relat ive flow velocity $U_{\infty}$, is the composition of the transverse speed $\Omega r_{g}$ and the main flow velocity $U_{\infty}$.

The lateral force coefficient depends on the dynamic cable angle, $\gamma\left(\gamma=\sqrt{\beta^{2}+\delta_{c}^{2}}\right)$ but it can be further simplified based on the following considerations about the variation range of $\gamma$. The static cable angle in the swing configuration in a general case is $\delta_{c}>6^{\circ} \cong 0.1 \mathrm{rad}$, so that the dynamic cable angle is always $\gamma>0.1$, but the value of the sideslip $\beta$ depends on the type of motion: small amplitude oscillations (swinging) or circular-orbit autorotation.

For small amplitude swinging oscillations a typical value of $\beta$ can be obtained using values of parameters coming from some previous exploratory tests performed in order to get some rough order of magnitude information (Gómez-Ortega, 2018). This value is given by

$$
\beta \simeq \frac{\theta_{\max } 2 \pi f_{R} r_{g}}{U_{\omega}} \simeq \frac{0.05 \times 2 \pi \times 2 \mathrm{~Hz} \times 0.04 \mathrm{~m}}{1 \mathrm{~m} / \mathrm{s}} \cong 0.02
$$




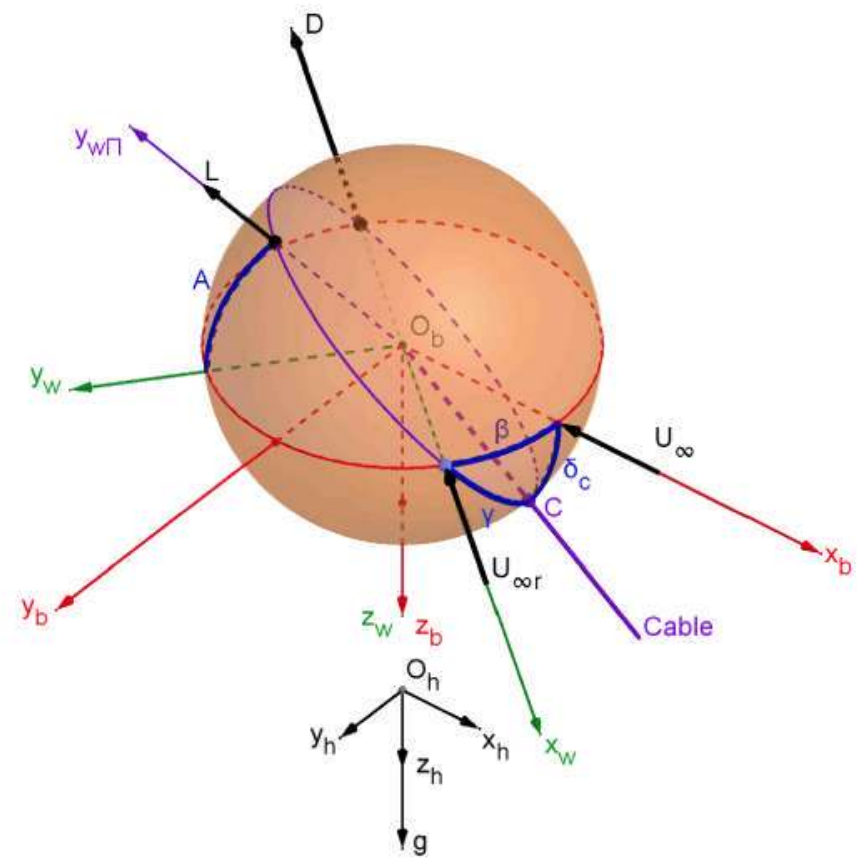

Fig. 4. Reference frames (as in Fig, 2) and aerodynamic forces, lift $L$ and $\operatorname{drag} D$. A: angle between the $x_{w}, y_{w}$ plane and $\Pi$ plane $\left(x_{w}, y_{w \pi}\right)$. Angles are represented using the unit radius sphere. Axonometric projection.

where $\theta_{\max }$ is the maximum amplitude angle of the oscillation, $f_{R}$ the pendulum frequency. Similar values of $\beta$ can be obtained for transmission line guard cables with beacons as both $r_{g}$ and $U_{\infty}$ are some 10 times larger than the values used in (12), and oscillation frequencies are close to $1.5 \mathrm{~Hz}$ (more tests should be performed to evaluate the generality of this result). Therefore, in this case, it can be considered $\beta \ll \delta_{c}$ and $\gamma$ can be assumed as almost constant, $\gamma \cong \delta_{c}$, and the expansions of $C_{L}(\gamma)$ and $C_{D}(\gamma)$ are given by

$$
\begin{aligned}
& C_{L}(\gamma)=C_{L}\left(\delta_{C}\right)+\left.C_{L \gamma}\right|_{\delta_{C}}\left(\gamma-\delta_{C}\right)+\cdots \cong C_{L}\left(\delta_{C}\right)=C_{L o} \\
& C_{D}(\gamma)=C_{D}\left(\delta_{C}\right)+\left.C_{D \gamma}\right|_{\delta_{c}}\left(\gamma-\delta_{C}\right)+\cdots \cong C_{D}\left(\delta_{C}\right) \simeq C_{D 0}
\end{aligned}
$$

From the above mentioned exploratory tests (Gómez-Ortega, 2018), it has been found that in the supercritical range the variations of $C_{L}(\gamma)$ and $C_{D}(\gamma)$ are small for small values of $\left(\gamma-\delta_{c}\right)$ and therefore the approximations (13) are valid. As the aerodynamic drag of the sphere has also been found to be almost independent of the cable angle, for small values of the cable angle, it can be assumed to have the same value as the isolated sphere case $C_{D 0}$.

In the case of a single sphere in a swing configuration but in circular-orbit autorotation, the angular rotation speed is $\Omega=2 \pi f \simeq 2 \pi \cdot 3.5 f_{R}$, where $f \cong 3.5 f_{R}$ from Gómez-Ortega et al. (2019), and $f_{R}$ is the natural frequency, therefore,

$$
\beta=\frac{2 \pi f r_{g}}{U_{\infty}}=\frac{2 \pi \times 3.5 \times 2 \mathrm{~Hz} \times 0.04 \mathrm{~m}}{10 \mathrm{~m} / \mathrm{s}}=0.17 \simeq 10^{\circ}
$$

and as in this case the rotation speed is a constant, $\beta$ and $\delta_{c}$ are constant values then also $\gamma_{a r}=\sqrt{\beta_{a r}^{2}+\delta_{c}^{2}}$ is a constant, therefore

$$
C_{L}=C_{L}\left(\gamma_{a r}\right)=C_{\text {Lar }} \text { and } C_{D}=C_{D}\left(\gamma_{a r}\right) \simeq C_{D 0}
$$

We discuss these two problems (small amplitude swinging oscillations and circular-orbit autorotation) in the following sections.

In this aerodynamic model, it is assumed that lift and drag are placed inside the symmetry plane of the relative wind (valid for the supercritical case behavior $\mathrm{Re}>\mathrm{Re}_{\mathrm{c}}$ ). The role of the unsteady forces generated by vortex shedding are not considered, as in the supercritical range the measured power spectra for the transverse forces does not show a strong peak at the frequency corresponding to the Strouhal number (Norman and McKeon, 2011a,b). In addition to that, autorotation, which is basically a steady motion, could not be explained by an unsteady oscillatory phenomenon (as vortex shedding). The same supercritical behavior appears in the subcritical range if the rotation speed is large enough, according to Norman et al. (2011), who studied the case of a protrusion rotating over a sphere. The projection of the aerodynamic forces (which are placed inside the symmetry plane of the relative flow) along the tangential direction of the motion, gives rise to a lateral force that can be responsible of the motion. 


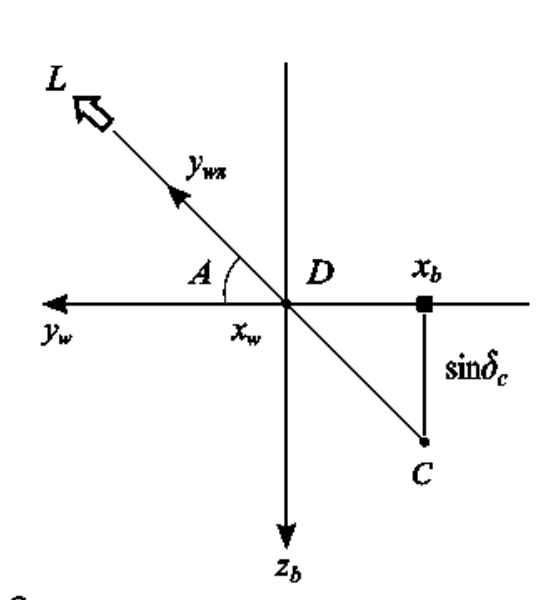

a
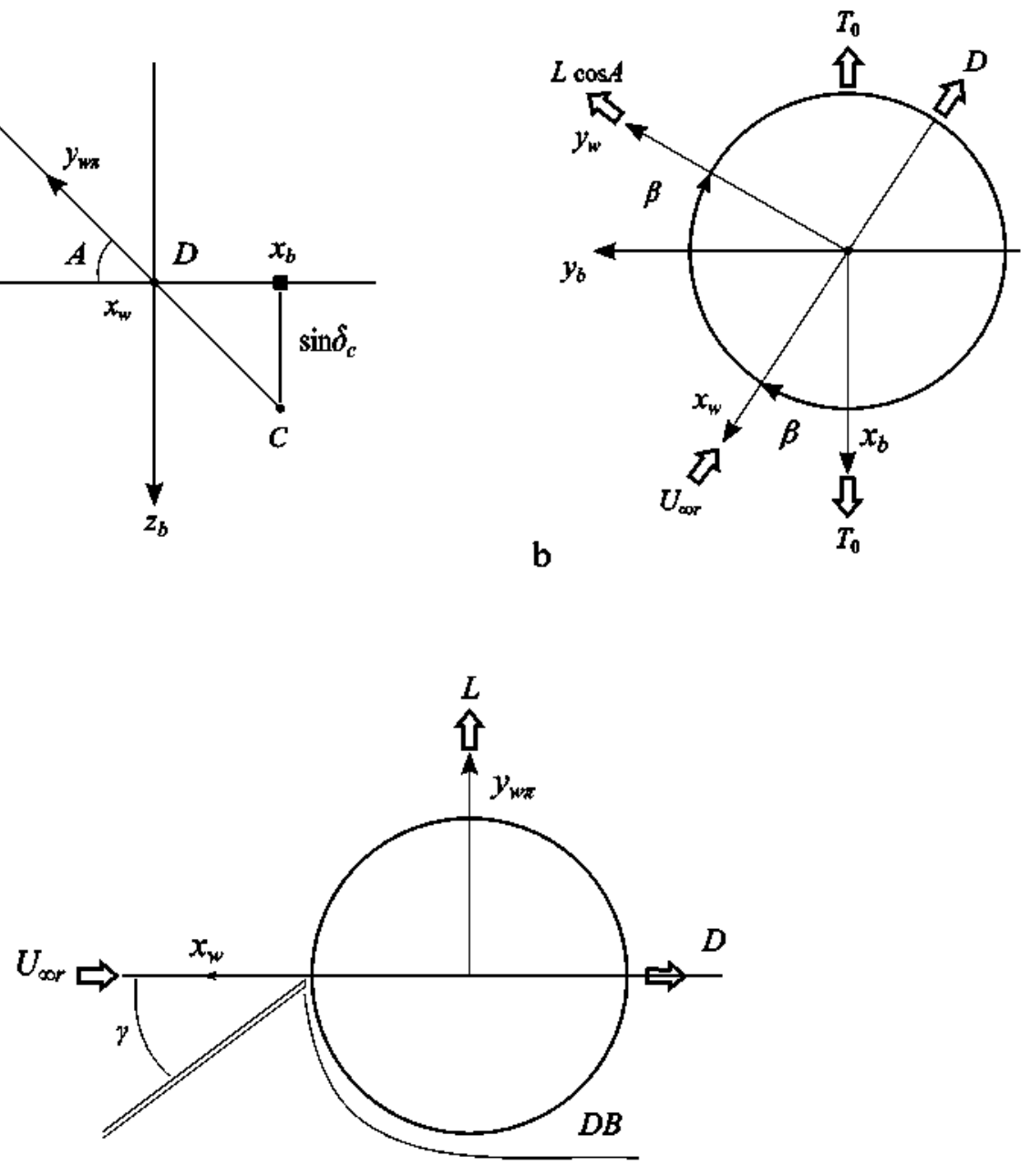

c

Fig. 5. Several projections of Fig. 4. (a) side view from $x_{w}$ axis ( $\Pi$ plane is seen from an edge, (b) Plan view from $-z_{w}$ axis ( $x_{b}, y_{b}$ plane), (c) Projection onto $\Pi$ plane $\left(x_{w}, y_{w \pi}\right.$ plane $)$. The detached boundary layer is outlined (DB).

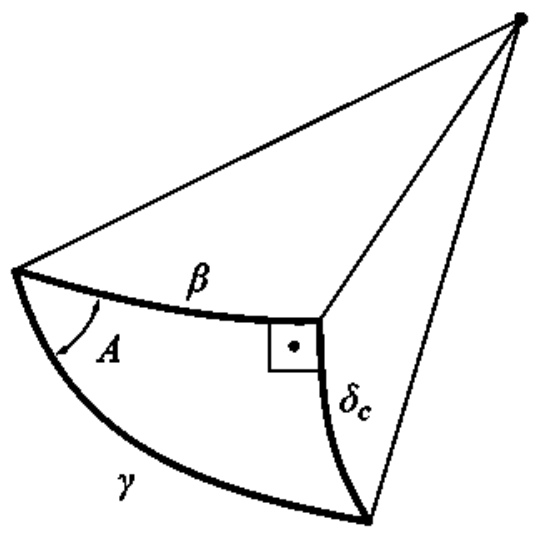

Fig, 6. Right spherical triangle. 


\section{Dynamic model for the transverse oscillation of a swinging sphere.}

The aerodynamic loads on the surface of a sphere in motion along a transverse direction have been obtained in Section 2. They can be applied to study the case of the small oscillations of a swinging sphere (Fig. 1b). The motion equation in the lateral direction $y$ can be derived from the model in (Gómez-Ortega, 2018)

$$
\ddot{y}-c \dot{y}+\omega_{g}^{2}\left(1+K_{L}\right) y=0
$$

where

$$
c \dot{y}=\frac{F_{L}}{m_{m}}, \omega_{g}^{2}=\frac{m_{T}}{m_{m}} \frac{g}{r_{g}} \text { and } K_{L}=\frac{L}{m_{T} g}=\frac{\frac{1}{2} \rho_{a} U_{\infty}^{2} S_{S} C_{L}}{m_{T} g} \ll 1
$$

and $\omega_{g}$ is the characteristic angular speed; $K_{L}$ the Tachikawa number, so-called load factor (Sanz-Andres and NavarroMedina, 2010); $\rho_{a}$ the air density; $m_{m}=m_{B}+m_{C} r_{P}+m_{A}$ is the moving mass, a combination of the beacon mass, $m_{B}$, the cable mass, $m_{C}$, and the air inertia additive mass, $m_{A}$ (which depends on the geometry of the moving body), $r_{P}$ is the participation coefficient, $m_{A}$ can be neglected in this case; $m_{T}=m_{B}+m_{C} / 2$ is the net mass affected by gravity forces (cable mass only contributes as $1 / 2) ; g$ gravity acceleration. The lateral force $F_{L}$ is given by (5)

$$
F_{L}=F_{y b}=L \cos A \cos \beta-D \sin \beta
$$

In the case of a swinging sphere, the sideslip angle is $\beta \gg 1$, therefore $\beta \simeq \frac{\dot{y}}{U_{\infty}}$ and the lateral force, using (11) and (13), can be written as

$$
F_{L}=\frac{1}{2} \rho_{a} U_{\infty}^{2} S_{S} \beta\left(\frac{C_{L}}{\gamma}-C_{D}\right)=\frac{1}{2} \rho_{a} U_{\infty}^{2} S_{S}\left(\frac{C_{L o}}{\gamma}-C_{D 0}\right) \frac{\dot{y}}{U_{\infty}}=m_{m} C_{0} C_{H} \dot{y}
$$

where $\gamma \simeq \delta_{c}$ as above mentioned. In this first approach, cable drag has not been considered for small amplitude oscillations. The damping parameter $c_{0}$ is given by

$$
c=c_{0} C_{H} \quad c_{0}=\frac{1}{2} \rho_{a} \frac{U_{\infty} S_{S}}{m_{m}} \quad C_{H}=\frac{C_{L O}}{\gamma}-C_{D 0}
$$

where $C_{H}$ is a kind of Den Hartog parameter, usually defined as $C_{L \alpha}+C_{D 0}$. Using the dimensionless transverse position $Y(t)$, and the complex amplitude $Y_{0}(\omega)$ defined as follows

$$
y=Y d_{B} \quad Y(t)=Y_{0}(\omega) \mathrm{e}^{\mathrm{i} \omega t}
$$

the oscillator equation (15) can be rewritten as

$$
\ddot{Y}-c \dot{Y}+\omega_{K}^{2} Y=0 \quad\left(-\omega^{2}-i c_{0} C_{H} \omega+\omega_{K}^{2}\right) Y_{0}=0
$$

where the corrected oscillation frequency is given by $\omega_{K}^{2}=\omega_{g}^{2}\left(1+K_{L}\right)$. Introducing the dimensionless frequency $\omega_{d}$ and damping coefficient $\zeta$ given by $\omega_{d}=\omega / \omega_{K}$ and $2 \zeta=c_{0} C_{H} / \omega_{K}$ the motion equation (21) can be rewritten in dimensionless form as

$$
-\omega_{d}^{2}-2 \mathrm{i} \zeta \omega_{d}+1=0
$$

which is the characteristic equation for a damped oscillator. The roots of the characteristic equation are

$$
\omega_{d}=-\mathrm{i} \zeta \pm \sqrt{1-\zeta^{2}}
$$

and the solution is (using the dimensionless time $\tau=\omega_{K} t$ )

$$
Y=Y_{0} \operatorname{expi}\left(-\mathrm{i} \zeta \pm \sqrt{1-\zeta^{2}}\right) \tau=Y_{0} \mathrm{e}^{\xi \tau}\left(\cos \sqrt{1-\zeta^{2}} \tau \pm \mathrm{i} \sin \sqrt{1-\zeta^{2}} \tau\right)
$$

The system is unstable for $\zeta>0$, that is, for $C_{H}>0$, that is $C_{L o} / \gamma>C_{D 0}$. Interestingly, this condition is similar (changing the sign) to the Glauert-Den Hartog for transverse galloping. The reason for the difference in sign is that the transverse component of the lift is oriented in the direction of the motion, while in the transverse galloping is oriented in the opposite direction.

The non-linear terms and the damping of the cable drag have not been considered, so that condition $C_{H}>0$ is only indicative of the start of the excited oscillations. Non-linear terms and cable drag will limit the oscillation amplitude.

As $\gamma \simeq \delta_{c}$, if $\delta_{c}$ decreases the unstable growth of oscillation amplitude should begin earlier (it is valid in the quasi-steady or supercritical regimes).

The transition from small amplitude oscillations to full circular-orbit autorotation (Gómez-Ortega et al., 2019) is very complex. The swinging oscillation motion at low speeds is produced by vortex induced vibrations (VIV), and as wind speed increases, after passing through several intermediate transition regimes, which depend on the value of the configuration parameters, the circular-orbit autorotation can be reached (see Section 4). Unfortunately, these transitions cannot be described with the help of a simple small amplitude planar pendulum model. A kind of chaotic motion appears, with intermittent intervals of different types of motions. 
Therefore, the results in this section can be applied to identify the parameters involved, their role in the onset of the instability, and an estimation of the instability boundary. The determination of the variation of the oscillation amplitude with wind speed or turbulent intensity is a more complex problem, to be considered in the light of experimental results.

\section{Dynamic model for circular orbit autorotation of a sphere in a swing configuration}

As explained in the previous section, as the wind speed increases, the small amplitude swing oscillation motion passes through some transition regimes and finally amplitude grows until the circular-orbit autorotation is established. As the final trajectory is a circle with a radius limited by the static equilibrium sag $r_{g}$, the mechanical description of the motion is greatly simplified (Fig. 1b). Besides, this motion appears at large wind speeds, leading to significant rotation speeds, so that gravity effects can be neglected compared to centrifugal forces (the motion seems to maintain a constant rotational speed). Thus, the mechanical problem is reduced to a simple condition: the tangential equilibrium is obtained when $C_{F y b}=0$ at sideslip $\beta=\beta_{a r}$, and therefore, from (11) and (14), the dynamic cable angle in autorotation, $\gamma_{a r}$ is given by

$$
\frac{C_{\text {lar }}}{\gamma}-C_{D 0}=0 \quad \gamma_{a r}=\frac{C_{\text {Lar }}}{C_{D 0}}
$$

This equilibrium condition is reached when the dynamic cable angle $\gamma_{a r}$ is equal to the lift-to-drag ratio $C_{L a r} / C_{D 0}$. The sideslip angle is

$$
\beta_{a r}=\sqrt{\gamma_{a r}^{2}-\delta_{C}^{2}}=\sqrt{\left(\frac{C_{L a r}}{C_{D 0}}\right)^{2}-\left(\frac{r_{g}}{l_{R}}\right)^{2}}
$$

and rotational speed around the support line SS (Fig. 1b) is given by

$$
\Omega_{a r}=\beta_{a r} \frac{U_{\infty}}{r_{g}}=\sqrt{\left(\frac{C_{L a r}}{C_{D 0}}\right)^{2}-\left(\frac{r_{g}}{I_{R}}\right)^{2}} \frac{U_{\infty}}{r_{g}}
$$

where $\Omega_{a r}$ is the equilibrium rotation speed at autorotation. As can be deduced from (27) this speed increases as the flow speed $U_{\infty}$ increases (as could be expected for an autorotation motion). The rotation radius $r_{g}$ has an opposite effect. The influence of the aerodynamic coefficients is limited to the lift coefficient $C_{I a r}$ and drag coefficient $C_{D O}$ obtained in representative static conditions. The influence of the geometrical parameters appears namely through the cable angle $\delta_{c}$.

The rotation speeds given by (27) using $C_{I a r}=0.1, C_{D 0}=0.4, \beta_{a r}=0.25$, are compatible with the above-mentioned preliminary experimental results obtained.

Concerning the flow itself, only the instantaneous sphere motion has been considered, but the full sweep of the orbit has not been taken into account, and the incoming flow has been considered uniform and quasi-steady. In these conditions it could be assumed that the cable wake is contained in the symmetry plane $\Pi$, and affects the sphere boundary layer starting at cable fixation point, and the introduced perturbation generates an asymmetry that is responsible for the lateral component of the lift force. However, if the cable rotation relative to the body is also taken into account, the cable wake will have a spiral shape when reaching the beacon. This spiral is created due to the delay introduced by the travel time between the cable and the beacon surface. This problem is left for a future study.

Besides, it should be considered that expressions (25)-(27) are just an approximation, as a result of the simplifications introduced (e.g. the absence of aerodynamic drag of the cable, a flat cable wake).

As above mentioned, in the case of circular-orbit autorotation of a sphere a in swing configuration, at large rotation speeds, the action of gravity has not been observed, which suggests that, along a complete turn the action of gravity force can be averaged to zero, although obviously gravity action affects the flow speed required to pass from swing oscillation to autorotation.

\section{Dynamic model for the circular-orbit autorotation of a hanging sphere under a vertical flow}

In this Section the dynamic model for the circular-orbit autorotation of a hanging sphere under a vertical flow is considered (Figs. 1a and 7), using the results for the aerodynamic loads obtained in Section 2.

The general motion of a hanging sphere under a vertical flow can be described as a spherical pendulum under gravity and aerodynamic forces. Once the flow velocity passes a given critical speed (Provansal et al., 2004) autorotation is excited and established, and in some cases a circular orbit is achieved. The motion is similar to the autorotation of a single sphere in a swing configuration (rotating the reference frame by $90^{\circ}$ around axis $y_{h}$ ), except that the effect of the gravity force in the hanging sphere case is not restrained by the trailing supporting wire (as in the sphere in a swing configuration) and therefore the instantaneous rotation radius $r_{g}$ is a free function of time that has to be determined as a part of the solution. This problem (without aerodynamic loads) can be traced back to the equilibrium of a rotating pendulum (Khan et al., 2013).

In order to get a first idea of the influence of the parameters involved, the simplest case has been considered, the circularorbit autorotation, where the equilibrium rotation radius $r_{g}$ is a constant to be determined.

The autorotation result for the tangential equilibrium (25) obtained in Section 4 can also be applied here, therefore $\beta$ $=\beta_{a r}$ but an additional condition, the radial equilibrium should be included. We consider the force component inside the 


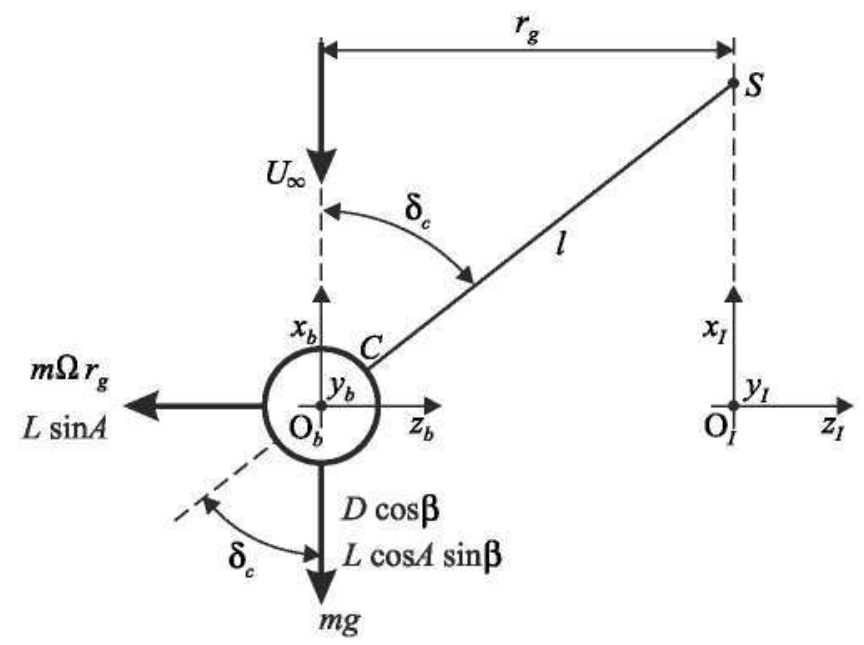

Fig. 7. Spherical pendulum under a vertical flow in circular-orbit autorotation around $x_{l}$ axis (Fig. 1a). Sketch of the equilibrium configuration. Force components in the meridian plane passing through the sphere center $\mathrm{O}_{b}$ and the rotation axis $x_{i},\left\{\mathrm{O}_{l}, x_{i}, y_{l}, z_{I}\right\}$ inertial reference frame. $S$ : cable support point; $C$ : sphere tether fixation point.

meridian plane and in the direction perpendicular to the cable's direction to get rid of the cable tension. The forces acting on the body, as shown in Fig. 7 are in radial equilibrium if the projection of the net force in the direction normal to the cable is zero

$$
\left(D \cos \beta+L \cos A \sin \beta+m_{B} g\right) \sin \delta_{c}=\left(m_{B} \Omega^{2} r_{g}+L \sin A\right) \cos \delta_{c}
$$

where $\beta=\beta_{a r}$ is given by (26), and the cable mass has been neglected. We are interested in the case $\beta, \delta_{c}, \ll 1$, therefore the following approximations can be used

$$
r_{g} \simeq 1 \delta_{c} \quad \sin A=\delta_{c} / \gamma \quad \cos A=\beta / \gamma \quad \cos \delta_{c} \simeq 1-\delta_{c}^{2} / 2
$$

where $l$ is the cable length. From (28) one obtains

$$
\left(D+L \frac{\beta}{\gamma} \beta+m_{B} g\right) \delta_{c}=\left(m_{B} \Omega^{2} I \delta_{c}+L \frac{\delta_{c}}{\gamma}\right)\left(1-\frac{\delta_{c}^{2}}{2}\right)
$$

where the second order term in the $\delta_{c}$ power series expansion of $\cos \delta_{c}$ has been retained.

Substituting the approximations (14) for $C_{L}$ and $C_{D}$, one obtains

$$
\left(q_{\infty} S_{S} C_{D O}+q_{\infty} S_{S} C_{\text {lar }} \frac{\beta^{2}}{\gamma}+m_{B} g\right)=\left(m_{B} \Omega^{2} l+q_{\infty} S_{S} \frac{C_{L a r}}{\gamma}\right)\left(1-\frac{\delta_{c}^{2}}{2}\right)
$$

Finally, using (25) expression (31) can be rewritten as

$$
\begin{aligned}
\delta_{c} & =\sqrt{2} \sqrt{1-\frac{q_{\infty} S_{S}\left(C_{D 0}+C_{L a r} \frac{\beta^{2}}{\gamma}\right)+m_{B} g}{m_{B} \Omega^{2} l+q_{\infty} S_{S} \frac{C_{a r r}}{\gamma}}} \\
& =\sqrt{2} \sqrt{\frac{m_{B}\left(\Omega^{2} l-g\right)+q_{\infty} S_{S}\left(\frac{C_{L a r}}{\gamma}-C_{D 0}-C_{L a r} \frac{\beta^{2}}{\gamma}\right)}{m_{B} \Omega^{2} l+q_{\infty} S_{S} \frac{C_{L a r}}{\gamma}}}=\sqrt{2} \sqrt{\frac{\frac{\Omega^{2}}{\Omega_{0}^{2}-1-\frac{q_{\infty} S_{S}}{m_{B} \Omega_{0}^{2} l} C_{D 0} \beta^{2}}}{\frac{\Omega^{2}}{\Omega_{0}^{2}}+\frac{q_{\infty} S_{S}}{m_{B} \Omega_{0}^{2} l} C_{D 0}}}
\end{aligned}
$$

which gives a relationship between the cable angle and the rotation speed, the configuration parameters and the aerodynamic coefficients. There is no solution of $(32)$ if the discriminant is negative.

To obtain some analytical expressions, let us define the dimensionless variables

$$
\Omega_{0}^{2}=\frac{g}{l} ; \quad \frac{\Omega}{\Omega_{0}}=1+\varepsilon_{\Omega}
$$

where $\Omega_{0}$ is the pendulum angular frequency, and minimum rotation equilibrium speed in the case of absence of aerodynamic forces. The combined influence of aerodynamic drag, reduced mass ( $m^{*}=m_{B} / m_{d}$, where $m_{d}$ is the displaced fluid mass), and geometrical parameters is condensed in parameter $\varepsilon_{1}$

$$
\varepsilon_{1}=\frac{q_{\infty} S_{S}}{m_{B} \Omega_{0}^{2} l} C_{D 0} \beta^{2}=\frac{3}{4 m^{*}} \frac{l}{d_{B}} C_{D 0} \delta_{c}^{2}
$$


For small values of $\delta_{c} \ll 1$ (the parameters involved should also be small $\varepsilon_{1}, \varepsilon_{\Omega} \ll 1$ ) from (32) one obtains

$$
\delta_{c} \simeq 2 \sqrt{\varepsilon_{\Omega}-\frac{\varepsilon_{1}}{2}}
$$

which can be used to deduce that for the autorotation to exist should be $\varepsilon_{\Omega}>0$, that is $\Omega>\Omega_{0}$ (angular rotation speed should be larger than the pendulum frequency), as usually is $\varepsilon_{1} \ll 1$. This is not the condition for the onset of autorotation, it is just the limit for a stable circular-orbit autorotation.

As expression (34) for $\varepsilon_{1}$ also contains the cable angle, (35) gives an implicit relationship between $\Omega$ and $\delta_{c}$.

As above mentioned, to solve the problem, the tangential equilibrium (25) must also be satisfied

$$
\gamma_{a r}=\frac{C_{L a t}}{C_{D D}}=\sqrt{\beta^{2}+\delta_{c}^{2}}=\delta_{c} \sqrt{1+\frac{1}{\mathrm{Fr}}\left(1+\varepsilon_{\Omega}\right)^{2}}
$$

which is obtained using both the expression for the sideslip and the dimensionless variable definition (33)

$$
\beta=\frac{\Omega r_{g}}{U_{\infty}} \simeq \frac{\Omega I}{U_{\infty}} \delta_{c}=\frac{\Omega_{0} I}{U_{\infty}}\left(1+\varepsilon_{\Omega}\right) \delta_{c}=\frac{1}{\sqrt{\mathrm{Fr}}} \delta_{c}\left(1+\varepsilon_{\Omega}\right)
$$

where $\mathrm{Fr}$ is the Froude number

$$
\mathrm{Fr}=\frac{U_{\infty}^{2}}{g !}=\left(\frac{U_{\infty}}{\Omega_{0} l}\right)^{2}
$$

and finally (taking into account that $\varepsilon_{\Omega} \ll 1$ )

$$
\delta_{c}=\gamma_{a r} \frac{1}{\sqrt{1+\frac{1}{\mathrm{Fr}}\left(1+\varepsilon_{\Omega}\right)}} \simeq \gamma_{a r} \frac{1}{\sqrt{1+\frac{1}{\mathrm{Fr}}}}
$$

In this first approximation, the equilibrium cable angle $\delta_{c}$ is independent of the rotation speed, it is only a function of the Froude number and $\gamma_{a r}$. The influence of the aerodynamic coefficients appear through the dynamic cable angle at autorotation $\gamma_{a r}$, given by (25), which implies that the effect of $C_{\mathrm{I} a t}$ and $C_{D 0}$ are in opposite directions, the first one helps to increase the cable angle, while the sphere drag reduces it. (39):

Two simple expressions can be obtained for the limit values of the static cable angle as a function of the Froude number

(a) Low flow speed $\mathrm{Fr} \ll 1$

$$
\delta_{c}=\gamma_{a r} \sqrt{\mathrm{Fr}}=\frac{C_{L a r}}{C_{\mathrm{DO}}} \frac{U_{\infty}}{g l} ; \quad \beta \simeq \gamma_{a r}
$$

the equilibrium cable angle $\delta_{c}$ linearly increases as flow speed increases, and decreases inversely proportional to the cable length. The slope of the linear variation is the dynamic cable angle (lift-to-drag ratio).

(b) Large flow speed, Fr $\gg 1$

$$
\delta_{c}=\gamma_{a r}\left(1-\frac{1}{2 \mathrm{Fr}}\right) ; \quad \beta \simeq \frac{1}{\sqrt{\mathrm{Fr}}} \gamma_{a r}
$$

showing that the equilibrium angle $\delta_{c}$ asymptotically tends towards a constant value $\gamma_{a t}$, which suggests that as flow speed increases the cable angle reaches a kind of saturation.

Therefore, at the first order approximation, the equilibrium angle $\delta_{c}$ can be obtained as an independent function of the rotation speed variation $\varepsilon_{\Omega}$. This value $\delta_{c}$ introduced in (35) and using $\varepsilon_{1}$ from (34), at first order leads to,

$$
\varepsilon_{\Omega}=\frac{\varepsilon_{1}}{2}+\frac{\delta_{c}^{2}}{4}=\frac{3}{811^{*}} \frac{l}{d_{B}} C_{D 0} \delta_{c}^{2}+\frac{\delta_{c}^{2}}{4}=\frac{1}{4} \delta_{c}^{2}\left[1+\frac{3}{2 m^{*}} \frac{1}{d_{B}} C_{D O}\right]
$$

which is the increment of rotation speed associated to a given equilibrium angle value $\delta_{c}$, In expanded notation

$$
\frac{\Omega}{\Omega_{0}}=1+\frac{1}{4} \delta_{c}^{2}\left[1+\frac{3}{2 m^{*}} \frac{l}{d_{B}} C_{D 0}\right]
$$

which gives the rotation speed $\Omega$ as a function of the cable angle, the reduced mass, the geometric parameter ratio, and the drag coefficient. Under the assumptions of the model, the effect of the sphere drag is to increase the rotation speed required for autorotation to achieve a given cable angle, in comparison with the rotation in vacuum conditions. The cortection term in (43) is usually small (as $\mathrm{m}^{*}$ is a large number) but for hollow spheres of light materials $\mathrm{m}^{*}$ can be small. The geometric parameter ratio is also a large number, therefore a significant correction term can be achieved. The correction term does not depend on the lift coefficient.

As $\delta_{c}=O\left(\gamma_{a r}\right) \ll 1$ the correction of the rotation speed is small even for large flow speeds.

The previous results can be compared to the experimental results of Provansal et al. (2003, 2004), where a configuration similar to Fig. 1a was considered. Provansal's experiments were conducted in a vertical water channel with a circular test 
section of diameter $8 \mathrm{~cm}$ and of length $100 \mathrm{~cm}$. The experimental results from Provansal that are relevant to the autorotation problem are the reduced frequency of the revolving motion (Fig. 19a of Provansal's paper) and the trajectory of the sphere (almost circular orbit, Fig. 19b). From Provansal's data the rotation rate $\Omega$ and the cable angle (or circular orbit radius) can be obtained and compared to the theoretical results.

In Provansal's tests glass spheres of diameter $d_{B}=16.6 \mathrm{~mm}$ (Reynolds numbers in the range 600-800) were used. A constant rotation rate was measured (Fig. 19a of Provansal's paper), the same as the natural frequency of the pendulum, independent of the flow speed, in the range tested, in agreement with the result of the model $\Omega \simeq \Omega_{0}$ from (43). From the data in Provansal's paper, the following parameters can be determined. As shown in Fig. 19b of Provansal's paper, the trajectory is a nearly-circular orbit, close to an ellipse with $2 \mathrm{~mm}$ and $1.6 \mathrm{~mm}$ semi-major and semi-minor axes, respectively. To simplify the process a mean radius $r_{\mathrm{g}}=1.8 \mathrm{~mm}$ is considered. The mass ratio is

$$
\mathrm{m}^{*}=\frac{m_{B}}{m_{t}}=\frac{\rho_{S}}{\rho_{a}}=\frac{2430}{1000}=2.43
$$

ln order to calculate the natural pendulum frequency, the value of the gravity $g$ to be considered should be modified taking into account the influence of buoyancy force and the change in the inertia due to the added mass. These influences are included in the apparent gravity acceleration g' (Provansal et al., 2004), given by

$$
g^{\prime}=\frac{m^{*}-1}{m^{*}+0.5} g=0.5 g \simeq 5 \mathrm{~m} / \mathrm{s}^{2}
$$

As in this experiments the pendulum length to sphere diameter ratio is $l / d_{B}=9$, the pendulum length is $l=9 d_{B}=$ $9 \times 16.6 \mathrm{~mm} \simeq 150 \mathrm{~mm}$, and the natural frequency is

$$
f_{R}=\frac{1}{2 \pi} \sqrt{\frac{g^{i}}{l}}=\frac{1}{2 \pi} \sqrt{\frac{5}{0.150}}=0.92 \mathrm{~Hz}
$$

This motion is observed at a reduced speed $U^{*}=4$, therefore the flow speed can be obtained from the reduced speed definition

$$
U_{\infty}=U^{*} f_{R} d_{B}=4 \times 0.92 \mathrm{~Hz} \times 0.0166 \mathrm{~m}=0.061 \mathrm{~m} / \mathrm{s}
$$

and the Froude number is $\mathrm{Fr}=\frac{u_{i 0}^{2}}{\mathrm{~g}^{i}}=\frac{(0.061)^{2}}{5 \backslash 0.15} \simeq 5 \times 10^{-3}$, thus $\sqrt{\mathrm{Fr}}=0.07$. Therefore, in this experiment the low speed range expression (40) can be used, together with (25), to obtain the value of the cable angle

$$
\delta_{c M}=\gamma_{a r} \sqrt{\mathrm{Fr}}=\frac{C_{l a r}}{C_{D 0}} \sqrt{\mathrm{Fr}}=\frac{0.12}{0.5} \times 0.07=0.017
$$

while the experimental result is $\delta_{C E} \simeq \frac{\mathrm{r} X}{i} \cong \frac{1.8 \mathrm{~mm}}{150 \mathrm{~mm}}=0.012$.

In the previous results, the values $C_{\text {Lat }}=0.12$ and $C_{D 0}=0.5$ have been used for the lift of a sphere with a cable, and for the sphere drag. An uncertainty of some $20 \%$ could be assigned to these values, and therefore the combined uncertainty could reach a $30 \%$. These values have been obtained from experiments at large Reynolds numbers (Gómez-Ortega, 2018) in static conditions. It should be pointed out that, according to Norman and McKeon (2011b), the experimental results obtained in supercritical conditions should also be valid for the unsteady subcritical conditions of Provansal's tests, although it needs confirmation from suitable experimental results, in representative conditions, which seems not to be a very simple exercise, as it should also consider the effect of the tether cable.

Anyway, taking into account the simplifications introduced in the mathematical model, and the possible issues associated to the experimental set-up (Reynolds number effects, blockage of the test section and wall effects in the sphere motion) the agreement between the model and experimental results, $\delta_{C M}$ and $\delta_{C E}$, respectively, is compatible with the uncertainty derived from the experimental data. This relative agreement gives us support on one hand to continue the work trying to obtain a better determination of the parameters involved in the mathematical model, and on the other hand to devote additional efforts to include further effects in this model, such as the cable drag and non-linear effects.

\section{Conclusions}

In this paper the revolving motion of a sphere about an axis which is eccentric with regard to the sphere central axis, under the action of a longitudinal flow (parallel to the rotation axis), has been considered as a model for the dynamics of circular-orbit autorotation or swinging oscillations that appears in several configurations, among others:

- Hanging sphere (spherical pendulum) under a vertical flow.

- Single sphere in a swing configuration (2D pendulum) under a horizontal flow.

- Multiple spheres in a swing configuration under a horizontal flow.

The two first configurations have been studied here. A model for the aerodynamic forces has been developed, which is applicable to these configurations. With the help of this aerodynamic model, simple dynamic models have been derived for the two kinds of motions. 
Concerning the dynamic model developed for the oscillation of a swinging sphere, it allows us to obtain the condition for the instability onset, $C_{L o} / \gamma-C_{D D}>0$. Therefore, as the dynamic cable angle is $\gamma \simeq \delta_{c}$, the cable angle should be smaller than the lift-to-drag ratio $\delta_{C}<C_{b o} / C_{D D}$. Interestingly, this parameter is similar to the one involved in the Den-Hartog condition for transverse galloping $C_{L \alpha}+C_{D 0}<0$.

Concerning the dynamic model developed for the circular-orbit autorotation motion, studied as a steady motion, conditions have been derived for the equilibrium configuration in the case of a single sphere both in a swing configuration and a hanging configuration (spherical pendulum).

For the autorotation of a single sphere in a swing configuration, only one equilibrium condition (the tangential force equilibrium) is needed, which allows us to obtain an expression for the angular rotation speed as a function of the lift and drag aerodynamic coefficients, the cable angle (which is fixed in this configuration) and the flow speed $U_{\infty}$.

But in the case of the spherical pendulum in autorotation, as it has two degrees of freedom, an additional equilibrium condition (the radial equilibrium) has to be included to obtain the equilibrium solution. In the case of a small cable angle, approximate expressions have been obtained for the cable angle and the rotation speed. In these expressions, the contributions of the relevant aerodynamic parameters of the sphere-cable system can be identified, as the lift and drag coefficients $C_{L}, C_{D 0}$, respectively, flow speed (through Froude number), and the geometric parameters.

Comparison of these results with experimental results from (Provansal et al., 2004; Gómez-Ortega et al., 2019; GómezOrtega, 2018) shows a relatively good agreement, taking into account the number of assumptions made in order to maintain the complexity of the model in a level as low as suitable for the purpose. The agreement level is giving support for us to continue with the work to consider the introduction of new effects in the dynamic model (cable drag, non-linear aerodynamic effects) and to obtain further experimental results, to check the results of the mathematical model, as far as possible, due to the limitations arising from the complexity of the tests.

\section{Acknowledgments}

The work presented in this paper is a part of the A3c2 project, which is financed by the Ministry of Science, Innovation and Universities of Spain (Ministerio de Ciencia, Innovación y Universidades, project reference: ENE2016-80107-R).

\section{References}

Achenbach, E., 1972. Experiments on the flow past spheres at very high Reynolds numbers. J. Fluid Mech, 54, 565-575.

Achenbach, E., 1974a. Vortex shedding from spheres. J. Fluid Mech. 62, 209-221.

Achenbach, E., 1974b. The effects of surface roughness and tunnel blockage on the flow past spheres. J. Fluid Mech, 65, 113-125.

Bacon, D.L., Reid, E.G., 1924. The resistance of spheres in wind tunnels and in air, Nat. Adv. Com. Aero. Rep. 185, Langley Memorial Aeronautical Laboratory, Langley.

Choi, J., Jeon, W-P., Choi, H., 2006. Mechanism of drag reduction by dimples on a sphere. Phys. Fluids 18, 041702. http://dx.doi.org/10.1063/1.2191848.

Etkin, B., 1972. Dynamics of Atmospheric Flight. John Wiley and Sons, Inc, New York.

Gómez-Ortega, O., 2018. (private communication).

Gómez-Ortega, O., Landeira, M., Ogueta-Gutiérrez, M., Franchini, S., Sanz-Andres, A., Chimeno, M., Roibás-Millán, E., Garcia-Perez, A., 2019. Transverse aeroelastic instability of guard cables with beacons excited by a longitudinal wind. J. Fluids Struct. 84, 122-139. http://dx.doi.org/10.1016/j.jfluidstructs. 2018.10.003.

Govardhan, R.N., Williamson, C.H.K., 2005. Vortex-induced vibrations of a sphere.J. Fluid Mech. 531, 11-47. http://dx.doi.org/10.1017/S0022112005003757.

Khan, Najeeb-A., Khan, Najeeb-A., Riaz, F., 2013. Dynamic analysis of rotating pendulum by hamiltonian approach. Chin. J. Math. 4. http://dx.doi.org/10. $1155 / 2013 / 237370$.

Lugt, H.J., 1983. Autorotation. Annu. Rev. Fluid Mech. 15, 123-147, http://dx,doi,org/10,1146/annurev.fl.15.010183.001011.

Norman, A.K., Kerrigan, E.C., McKeon, B.J., 2011. The effect of small amplitude time-dependent changes to the surface morphology of a sphere. J. Fluid Mech. 675, 268-296. http://dx.doi.org/10.1017/S0022112011000164.

Norman, A.K., McKeon, B.J., 2011a. The effect of a small isolated roughness element on the forces on a sphere in uniform flow. Exp. Fluids 51, $1031-1045$. http://dx.doi.org/10.1007/s00348-011-1126-y.

Norman, A.K., McKeon, B.J., 2011b. Unsteady force measurements in sphere flow from subcritical to supercritical Reynolds numbers. Exp. Fluids 51,1439 1453. http://dx,doi.org/10.1007/s00348-011-1161-8.

Provansal, M., Leweke, T., Schouveiler L. Guébert, N., 2003. 3D oscillations and vortex induced vibrations of a tethered sphere in a flow parallel to the thread. In: Proc, of the 4th Pacific Symposium on Flow Visualization and Image Processing. Chamonix, France.

Provansal, M., Schouveiler, L., Leweke, T., 2004. From the double vortex street behind a cylinder to the wake of a sphere. Eur. J. Mech. B Fluids $23,65-80$. http://dx.doi.org/10.1016/j.euromechflu.2003.09.007.

Riabouchinsky, D.P., 1935. Thirty years of theoretical and experimental research in fluid mechanics. J. R. Aeronaut. Soc. 39, 282-348, $377-444$.

Sanz-Andres, A., Navarro-Medina, F., 2010. The initiation of rotational motion of a lying object caused by wind gusts. J. Wind. Eng. Ind. Aerodyn. $98,772-783$. http://dx.doi.org/10.1016/j.jweia,2010.07.002.

Wertz, J.R., Larson, W., 1999. Space Mission Analysis and Design. Springer Netherlands, ISBN: 978-0-7923-5901-2.

Williamson, C.H.K., Govardhan, R., 1997. Dynamics and forcing of a tethered sphere in a fluid flow. J. Fluids Struct. 11, 293-305.

Williamson, C.H.K., Govardhan, R., 2008. A brief review of recent results in vortex-induced vibrations. J. Wind Eng. Ind. Aerodyn. 96, 713-735.

Willmarth, W.W., Enlow, R.L., 1969. Aerodynamic lift and moment fluctuations of a sphere. J. Fluid Mech. 36, 417-432. 